\title{
Calculating Compressive and Flexural Strength of Concrete by Computer Program.
}

\author{
Santosh G Gandevikar, M Tech (Structure) Student, Ganpat University, India. \\ santoshg.str@gmail.com \\ Prof. Arjun M Butala, Department of Civil Engineering, Ganpat University, India, \\ arjunbutala@gmail.com
}

\begin{abstract}
The testing of concrete for its desired strength after casting is one of prime activities quality control of civil engineering project executions. The concrete is chiefly tested for its compressive strength and then the results are compared or checked with the desired design strength. In the era of computers used in every spectrum of life, the testing of materials and its results calculations also needs to be programed for calculations, validations and data storage. The cell based software do provide ease of calculations but storage and retrieval of data is difficult in those software. The current study is an effort to develop a program the calculations of compressive strength of concrete and flexural strength of the concrete as per Indian Standard Code of Practice. The standalone customized software used in calculations gives accurate and consistent results. Also, the validations programed with logics avoid hazy manual interpretations of the regulatory provisions and gives accurate results.
\end{abstract}

Key Words : Compressive Strength, Computer Program, Flexural Strength.

\section{INTRODUCTION}

Cement concrete is one of the major materials of construction in the present times. Concrete is used more than any other man-made material on the planet. There is about 6 billion cubic meters of concrete are made each year, which equals one cubic meter for every person on Earth. Also, it is the only material that can be used for any kind of civil engineering structure. Due to its versatility in manufacturing, handling, transportation, casting to any strength, it is the material of preference where strength, performance, durability, impermeability, fire resistance, abrasion and rot resistance are required. Cement concrete though apparently simple but actually complex material, due to its changing form from dry to wet, plastic and then hardened with chemical reactions and environmental conditions. Hence to achieve desired strength of the concrete, testing of concrete plays an important role in controlling and confirming the quality of cement concrete works. Systematic testing of the raw materials, fresh concrete and hardened concrete are indispensable part of any quality control program for civil engineering project. These helps to achieve higher efficiency of material used and grater assurance of the performance of the concrete with regard to both strength durability. Different regulatory authorities / bodies specify criteria for strength and testing as per the requirement of the particular zone / country. Indian Standard Test procedure[1] are adopted in the current study testing of concrete.
The concrete be tested at two stages, (i) Fresh Concrete and (ii) Hardened Concrete. Fresh concrete or plastic concrete is freshly mixed cement, aggregates, water and / or admixtures, ready for casting in to any shape. The predetermined quantities ascertained by mix design are mixed together and controlled the properties of concrete in the wet state, till pouring or casting. The fresh concrete is tested for workability (Slump Test), bleeding (Bleeding Test) and segregations (Vee-Bee Test).

The purpose of testing hardened concrete is to confirm that the concrete used at site has developed the required the desired strength. As the hardening of the concrete takes time it is not possible to know the actual strength of the concrete for some time. Also being mixture of different materials acted by chemical reactions the strength prediction or assurance of strength of structural concrete component is difficult by direct method. Instead indirect methods like cubes or cylinders casted during the actual pouring of the concrete are tested for various strength parameters and the strength of the structural components is thus known. Generally, the hardened concrete is tested for density, compressive strength, flexural strength, tensile strength, modulus of elasticity, permeability, cement content etc. Out of above list tests compressive strength and flexural strength very important and essentially carried out as part of concrete testing. 


\section{(A) Compressive Strength Test:}

Indian Standard Code of Practice - IS 516 : 1959 Reaffirmed 2013[1], states various methods for testing of concrete. The compressive strength of the concrete is found out as per clause 5 of the IS 516 . In this method concrete cube of $15 \mathrm{~cm}$ $\mathrm{x} 15 \mathrm{~cm} \times 15 \mathrm{~cm}$ or cylinders of $15 \mathrm{~cm}$ diameter and $30 \mathrm{~cm}$ length are tested for compressive strength. Test samples at recognized ages of 7 and 28 days or any other period as specified first kept in water at for 24 hours at a temperature between $24^{\circ}$ to $32^{\circ}$. After 24 hours the samples taken from water, wiped off and then immediately placed in compression testing machine. Where it may be necessary to obtain the early strengths, tests are made at the ages of 24 hours $\pm 1 / 2$ hour and 72 hours \pm 2 hours of age of concrete.

The sample is then given vertical load at approximate rate of $140 \mathrm{~kg} / \mathrm{sqcm} / \mathrm{min}$. The final load at failure is recorded. The load divided bearing area of the cube gives compressive strength of the cube. The results are reported by checking the general criteria for 7 days (67\% of specified strength) and 28 days( $100 \%$ of specified strength) table-1. Also, individual compressive strength for a cube out of set of three cubes shall not have variation of results by $\pm 15 \%$ than the average else the results. The cube strength of the cylindrical sample be obtained by multiplying 1.25 times the cylindrical compressive strength.

Table-1. Compressive Strength of Concrete at various age.

\begin{tabular}{|c|c|c|}
\hline $\begin{array}{l}\text { Grade of } \\
\text { Concrete }\end{array}$ & $\begin{array}{c}\text { Minimum } \\
\text { compressive strength } \\
\mathrm{N} / \mathrm{mm}^{2} \text { at } 7 \text { days }\end{array}$ & $\begin{array}{c}\text { Specified characteristic } \\
\text { compressive strength } \\
\mathrm{N} / \mathrm{mm}^{2} \text { at } 28 \text { days }\end{array}$ \\
\hline M15 & 10.0 & 15.0 \\
\hline M20 & 13.5 & 20.0 \\
\hline M25 & 17.0 & 25.0 \\
\hline M30 & 20.0 & 30.0 \\
\hline M35 & 23.5 & 35.0 \\
\hline M40 & 27.0 & 40.0 \\
\hline M45 & 30.0 & 45.0 \\
\hline
\end{tabular}

\section{(B) Flexural Strength Test:}

As per clause 8 of Indian Standard Code of Practice - IS 516 : 1959 Reaffirmed 2013[1], the flexural strength of the concrete can be determined by two-point test. In this method either of two sample sizes, immediately on removal from the water whilst they are still in a wet condition. The dimensions of each specimen shall be noted before testing. The test specimen in placed in loading arrangement as per figure- 1 . Two sizes either $10 \mathrm{~cm} \times 10 \mathrm{xcm} \times 50 \mathrm{~cm}$ or $15 \mathrm{~cm} \times 15 \mathrm{~cm} \times 70 \mathrm{~cm}$ is tested for flexural strength. The sample size $10 \mathrm{~cm} x 10 \mathrm{xm} \times 50 \mathrm{~cm}$ is used when maximum size of the aggregates used in $19 \mathrm{~mm} .15 \mathrm{~cm} \times 15 \mathrm{~cm} \times 70 \mathrm{~cm}$ size is used for aggregates size is more than $19 \mathrm{~mm}$ used in concrete. Test specimens kept immersed in water at a temperature of $24^{\circ}$ to $30^{\circ} \mathrm{C}$ for 48 hours before testing, and tested While The load is applied without shock and increasing continuously at a rate at a rate of loading of $400 \mathrm{~kg} / \mathrm{min}$ for the $15.0 \mathrm{~cm}$ specimens and at a rate of $180 \mathrm{~kg} / \mathrm{min}$ for the $10.0 \mathrm{~cm}$ specimens. The load is increased until the specimen fails, and the maximum load applied to the specimen during the test is recorded. Also, distance between crack appeared on tension face of the specimen and nearest support is noted down for calculation purpose.

figure-1.
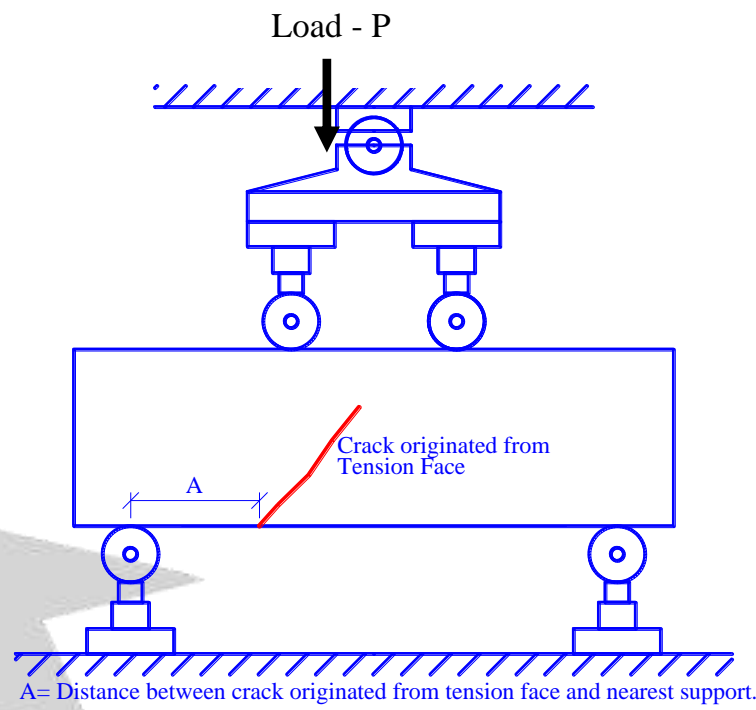

Figure -1. Flexural Test Arrangement

The flexural strength fb of the specimen is calculated using following formula, asper IS-516[1]

(1) If ' $\mathrm{A}$ ' is greater than $20.0 \mathrm{~cm}$ for $15.0 \mathrm{~cm}$ specimen, or greater than $13.30 \mathrm{~cm}$ for a $10.0 \mathrm{~cm}$ specimen,

$$
f b=\frac{p \times l}{b \times d^{2}}
$$

(2) If ' $A$ ' is less than $20.0 \mathrm{~cm}$ but greater than $17.0 \mathrm{~cm}$ for $15.0 \mathrm{~cm}$ specimen, or less than

$$
f b=\frac{3 \times p \times l}{b \times d^{2}}
$$

Where $\mathrm{p}=$ failure load and $1, \mathrm{~b}, \mathrm{~d}$ are length, width and height of specimen respectively, $13.3 \mathrm{~cm}$ but greater than 11.0 $\mathrm{cm}$ for a $10.0 \mathrm{~cm}$ specimen

\section{II . CURRENT PROJECT}

\section{Methodology}

From the host of computer programming languages available in the market C\# pronounced as C Sharp is used for programing in the current study. C Sharp is a multiparadigm programming language that supports imperative, generic and object-oriented programming. It is a part of the Microsoft .NET Framework. It is similar to $\mathrm{C}++$ in its object-oriented syntax and is also influenced by Java and Delphi. The flexibility of C Sharp for desktop standalone application and web applications with static local data and dynamic server data is also merits for its section. 
The development of any computer program has five parts or divisions.

\section{Clarifying / Defining the Problem:}

In this part the objectives of the program, desired output, desired input, processing of input with methods, feasibility of the implementing the program as decided.

\section{Designing the problem.}

In this part the flow of the operation on input parameter and deriving output parameter is planned. In case of object oriented Graphic User Interface (GUI) based program, forms are designed to house the inputs and output are also designed. Flow charts with logics are prepared in this part and all the raw work made ready for the coding. The repeatedly used methods / parts of programs called as functions are also decided and defined in this part. The functions are named part of a program with or without return value.

\section{Coding the Program.}

In this parts forms with control like, textboxes, buttons, labels, combo / list boxes, radio buttons or any other type of user friendly \& understandable controls are prepared. Variable of appropriate type like, string, integer, single, double, Boolean etc. are assigned to the input and out put data. Arithmetic, trigonometrical operations with and without iterative actions are coded as per the syntax of the language. Errorless and correct results are ensured by putting intermediate checks and debugging commands. The final results with input data and output data if required is stored on local hard disk or server as per the requirement.

\section{Testing the Program.}

In this part the program coded as above is tested for its correctness and accuracy. The input data is entered in the forms and the output obtained is checked / verified / validated though other tools. Errors, ambiguities etc. traced in the program and removed / corrected and final correct output is obtained.

\section{Documentation.}

Once the coding of the program is established as correct one giving desired validated results the code is cleaned up for the unnecessary debug /checking points, duplicated variables, temporary sub programs etc. are streamlined and a neat and clean code is prepared and backed up as security.

\section{Programing in C Sharp.}

\section{(1) Clarifying and defining the problem.}

In this part two concrete tests viz compressive strength and flexural strength were selected for programming. The objectives to find out compressive strength ' $\mathrm{fc}$ ' and flexural strength ' $f b$ ' are determined. Also, the conformity as per the respective provisions of Indian standard code is fixed as objective. The input and output parameters like sizes of samples, grade of concrete, maximum load taken till failure etc. are defined as variables.

\section{(2) Designing the problem.}

Here chronology for the input data and its calculations is designed. For Compressive strength test input data like sample ID, Machine Constant, Failure Load Reading, Dimensions of the cube, curing period, grade of the concrete etc. data is used as input hence appropriate type of variables are decided for it. General validations for input data like preventing alphabet, symbol, null values, multiple decimal points etc. in to input text boxes are decided and coded accordingly. The input data is converted in decimal numbers from text and stored to various variables. Mathematical calculations, logical operations are planned in systematic manner so as to have correct results while coding. The repeatedly used operations which are called functions like, calculating volume, density of each specimen, calculating average of three specimen are planned in this part.

Logical operations for deciding the conformity with IS Code like if the individual strength of the specimen is differing more than $\pm 15 \%$ of the average compressive strength of three sample, the user be given to option either to discard the results and start data entry afresh or whatever the results are required to be stored is also formed in this part.

\section{(3) Coding the Program.}

\section{(A) Compressive Strength Calculations for Concrete Cubes}

The actual work of form design with programing is done in this part. The form for Compressive Strength of Concrete Cubes is designed considering data to be entered. Different names to variable are assigned on form and in code as per table-1.

The input on the form are defined by separate variable name than the code variable to avoid conflict or variables. FCCMCCon stands for Form Concrete Compressive

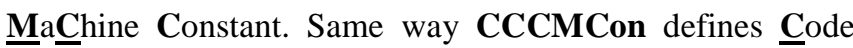

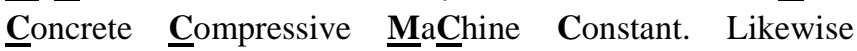
Length, Width, Height and Weight of three cubes is input on form and are stored in the code for further operations. The dimensional parameters are used to work out bearing area of the cubes and density of the cubes. The Machine constant is calibration constant for a particular compressive test machine to which observed load is multiplied to arrive at actual load. The actual load divided by bearing gives the compressive strength for each cube and average value of three cubes is reported as result. 
Table - 2 Defining Variables

\begin{tabular}{|c|c|c|}
\hline Variables & On Form & In Code \\
\hline Input & \multicolumn{2}{|c|}{ Open for Input, the input shall be non -zero, non-negative, non-alphabetic } \\
\hline Machine Calibration Constant & FCCMCCon & CCCMCCon \\
\hline Length of Sample & FCCLen1, FCCLen2, FCCLen3 & CCCLen1, CCCLen2, CCCLen3 \\
\hline Width of Sample & FCCWid1, FCCWid2, FCCWid3 & CCCWid1,CCCWid2, CCWid3 \\
\hline Height of Sample & FCCHt1, FCCHt2, FCCHt3 & CCCHt1, CCCHt2, CCCHt3 \\
\hline Weight of Sample & FCCWt1, FCCWt2, FCCWt3 & CCCWt1, CCCWt2, CCCWt3 \\
\hline Observed Load & FCCObR1,FCCObR2, FCObR3 & CCCObR1,CCCObR2, CCObR3 \\
\hline Concrete Grade & FCCGrade & CCCGrade \\
\hline Age in Days & $\begin{array}{l}\text { Rb07, Rb28 ( on group box only one can be selected } \\
\text { at a time ) }\end{array}$ & Not required \\
\hline Output & \multicolumn{2}{|c|}{ Locked for Input, } \\
\hline Density of Concrete & FCCDen1, FCCDen2, FCCDen3 & CCCDen1,CCCDen2, CCCDen3 \\
\hline Actual Load & FCCActR1,FCCActR2, FCCActR3 & CCCActR1,CCCActR2, CCCActR3 \\
\hline Compressive Stress & FCCCS1, FCCCS3, FCCCS3 & CCCS1, CCCS3, CCCS3 \\
\hline
\end{tabular}

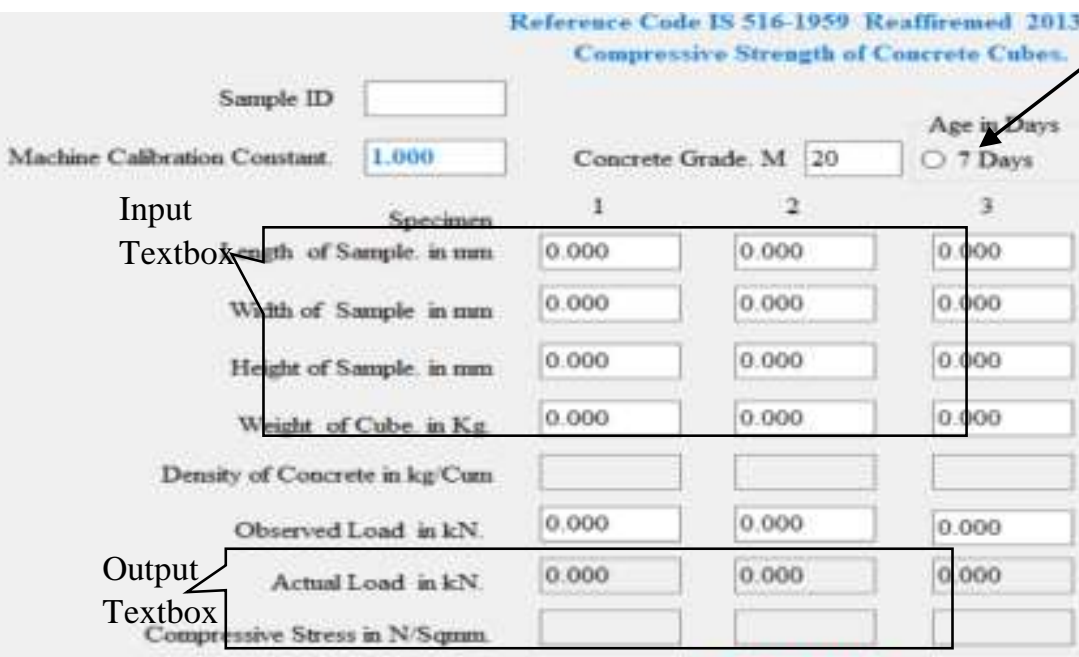

REMARKS

RFQUAIRED MINTMUI COMPRESSTVE STRENGTH FOR M 20 GRADF CONCRETE

AT 28 DAYS AGE OF CONCRETE = 20 NSqum.

Figure -2 Form Design for Compressive Strength Test

The figure-2 shows form design for Compressive Strength Test. The white coloured boxes are called textboxes and are used for data input. The gray coloured boxes are for the results and locked for editing so the possibility of mistakenly entering data in the results textboxes is eliminated. The general data like Sample ID, is entered and handled as string. The Machine Constant, Length of Sample, Width of Sample, Height of sample, Weight of Sample, Observed reading of failure load are stored on double type variables which in turn allows mathematical operations on it. The data entered on the form is always a string data from the textbox hence it needs to be converted in to double data type. The calculations of density are triggered and executed while leaving the textbox called Weight of Cube. The compressive strength is calculated as soon as leaving the textbox called Observed Reading.

Following is the code or actual program in C-Sharp. Here, first the repeatedly used tasks called as functions are defined. The functions reduces the length of the total program and speeds up the execution of the program. In compressive strength program average function is used for 3 sets of lengths, widths, heights, weight of cubes, observed readings, and compressive strengths. Similarly volume function calculates volume for as many samples but written only once.

The bold and italic text is explanation and normal text is the code.

\section{Comment statement doesn't participate in program.}

//compressive strength program

Defining the function of average of three numbers public double ThreeAvg(double a, double b, double c)

\{

double avg3 = Math.Round $((a+b+c) / 3,2)$; return avg3;

\}

\section{Defining the function of calculation of volume} public double $\mathrm{CCV}$ ol (double a, double b, double c) \{

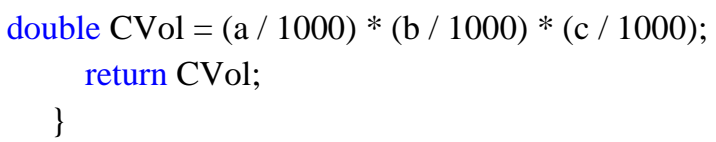

Data entry for sample Identification number

private void FCSSampleID_TextChanged(object sender, EventArgs e) 


\section{\{}

CCSSampleID = FBKCSSampleID.Text;

\}

FCCMCCon.Text is form input converted in to decimal bearing number for machine constant and stored as CCCMCon in code

private void FCCMCCon_Leave(object sender, EventArgs

e)

\{

CCCMCCon $=$ Convert. ToDouble $($ FCCMCCon. Text $)$;

\}

Form input for sample length, width and height are converted in to decimal bearing number and stored in code private void FCCLen1_Leave(object sender, EventArgs e)

\{

CCCLen $1=$ Convert.ToDouble(FCCLen1.Text $)$;

\}

e)

private void FCCLen2_Leave(object sender, EventArgs

CCCLen2 = Convert.ToDouble(FCCLen2.Text);

\}

private void FCCLen3_Leave (object sender, EventArgs e)

\{

CCCLen3 = Convert.ToDouble(FCCLen3.Text);

Function to calculate average is called the function is used for 3 times, viz for average of length, width and height. CCCLenAvg $=$ ThreeAvg(CCCLen1, CCCLen2,

CCCLen3);

FCCLenAvg.Text $=$ CCCLenAvg.ToString () ;<smiles>[Tl]</smiles>

private void FCCWid1_Leave(object sender, EventArgs

e)

CCCWid 1 = Convert.ToDouble(FCCWid1.Text);

\}

private void FCCWid2_Leave(object sender, EventArgs

e)

\section{\{}

CCCWid2 $=$ Convert.ToDouble $($ FCCWid2.Text $)$;

\}

private void FCCWid3_Leave(object sender, EventArgs

e)

\section{\{}

CCCWid3 = Convert.ToDouble(FCCWid3.Text);

CCCWidAvg $=$ ThreeAvg $(C C C W i d 1$, CCCWid2,

CCCWid3);

FCCWidAvg.Text = CCCWidAvg.ToString();

$$
\text { \} }
$$

private void FCCHt1_Leave(object sender, EventArgs e)

$$
\{
$$

$\mathrm{CCCH} 1 \mathrm{l}=$ Convert.ToDouble $(\mathrm{FCCH} 1$. Text $)$;

\}

private void FCCHt2_Leave(object sender, EventArgs e)

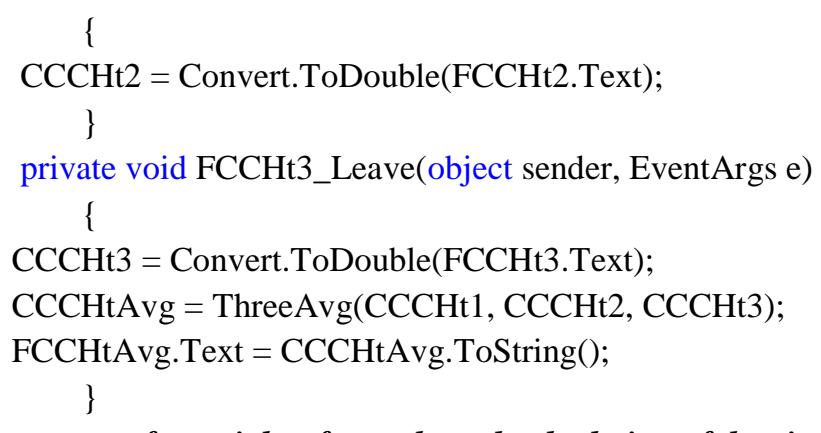

Data entry for weight of sample and calculation of density, also writing the density result on form after converting from double to string

Here function volume is called to calculate volume of three concrete cubes.

private void FCCWt1_Leave(object sender, EventArgs e)

CCCWt $1=$ Convert.ToDouble $($ FCCWt1.Text $)$;

CCCDen $1=$ Math.Round $($ CCCWt1 $/(C C V o l(C C C L e n 1$,

CCCWid1, CCCHt1))), 3);

FCCDen1.Text $=$ CCCDen1.ToString();

\}

private void FCCWt2_Leave(object sender, EventArgs e)

CCCWt $2=$ Convert. ToDouble $($ FCCWt2.Text $)$ CCCDen2 $=$ Math.Round((CCCWt2 / (CCVol(CCCLen2, CCCWid2, CCCHt2))), 3);

\section{FCCDen2.Tex}

CCCDen2.ToString();

\}

private void FCCWt3_Leave(object sender, EventArgs e)

$\mathrm{CCCWt} 3=$ Convert. ToDouble $(\mathrm{FCCWt} 3$. Text $)$;

CCCDen3 = Math.Round $((\mathrm{CCCWt} 3 /$ (CCVol(CCCLen3, CCCWid3, CCCHt3))), 3);

FCCDen3.Text $=$ CCCDen3.ToString () ;

Function to calculate average is called

CCCWtAvg $=$ ThreeAvg $(C C C W t 1$, CCCWt2, CCCWt3 $)$;

FCCWtAvg.Text $=$ CCCWtAvg.ToString();

CCCDenAvg $=$ ThreeAvg $($ CCCDen1, CCCDen2,

CCCDen3);

FCCDenAvg.Text $=$ CCCDenAvg.ToString () ;

\}

Data entry for observed load, calculating actual load and compressive strength for all three samples and writing it on the form

private void FCCObR1_Leave(object sender, EventArgs e)

\{

CCCObR1 $=($ Convert.ToDouble $($ FCCObR1.Text $)) ;$

CCCAct $1=$ Math.Round $((\mathrm{CCCMCCon} *$ CCCObR1 $), 2)$;

CCCCS1 $=$ Math.Round $((C C C A c t 1 * 1000) /($ CCCLen $1 *$

CCCWid1), 2);

FCCActR1.Text $=$ CCCAct 1 .ToString();

FCCCS1.Text $=$ CCCCS1.ToString () ;

$$
\text { \} }
$$

private void FCCObR2_Leave(object sender, EventArgs e) 
\{

CCCObR2 $=($ Convert.ToDouble $($ FCCObR2.Text $)) ;$

CCCAct $2=$ Math.Round $((\mathrm{CCCMCCon} *$ CCCObR2 $), 2)$;

CCCCS2 $=$ Math.Round $((C C C A c t 2 * 1000) /($ CCCLen $2 *$ CCCWid2), 2);

FCCActR2.Text $=$ CCCAct2.ToString () ;

FCCCS2.Text $=$ CCCCS2.ToString();

\}

private void FCCObR3_Leave(object sender, EventArgs e)

\{

CCCObR3 $=($ Convert.ToDouble $($ FCCObR3.Text $))$;

CCCAct $3=$ Math.Round $((\mathrm{CCCMCCon} *$ CCCObR3), 2);

CCCCS3 $=$ Math.Round $(($ CCCAct $3 * 1000) /($ CCCLen $3 *$

CCCWid3), 2)

Function to calculate average is called

CCCActAvg $=$ ThreeAvg (CCCAct 1, CCCAct2, CCCAct 3$)$;

CCCObRAvg $=$ ThreeAvg $(C C C O b R 1$, CCCObR2,

CCCObR3);

CCCCSAvg $=$ ThreeAvg $($ CCCCS1, CCCCS2, CCCCS3 $)$;

FCCActR3.Text $=$ CCCAct3.ToString () ;

FCCCS3.Text $=$ CCCCS3.ToString(); FCCObRAvg .Text $=$ CCCObRAvg.ToString();

FCCActRAvg. Text $=$ CCCActAvg. ToString();

FCCCSAvg.Text $=$ CCCCSAvg.ToString () ;

Checking individual compressive strength with respect to average compressive strength for variation of $+/-15 \%$ if $($ CCCCS $1<0.85 *$ CCCCSAvg $\|$ CCCCS $1>1.15 *$ CCCCSAvg $\|$ CCCCS2 $<0.85 *$ CCCCSAvg $\|$ CCCCS2 $>$ $1.15 *$ CCCCSAvg $\|$ CCCCS3 $<0.85 *$ CCCCSAvg $\|$ CCCCS3 $>1.15 *$ CCCCSAvg)

MessageBox.Show("Individual Values differ more than +/$15 \%$ of Average Compressive Values");

If the results are non-confirming then user is given option to re-test the sample or else non-confirming results are stored to record.

DialogResult ChangeVal $=$ MessageBox.Show("Individual Values differ more than $+/-15 \%$ of Average Compressive Values.....Do You Want to Change The Results", "Change Results", MessageBoxButtons.YesNo);

If the uses opts for discarding non-confirming tests results and wishes to start fresh data entry the old values are discarded by making input cell value $=0.000$

if (ChangeVal $==$ DialogResult.Yes)

\{

FCCLen1.Text $=$ "0.000";

FCCLen2.Text $=$ "0.000";

FCCLen3.Text $=$ "0.000";

FCCWid1.Text $=$ "0.000";

FCCWid2.Text $=$ "0.000";

FCCWid3.Text $=$ "0.000";

FCCHt1.Text = "0.000";

FCCHt2.Text $=" 0.000 "$;

FCCHt3.Text $=" 0.000 "$;

FCCWt1.Text = "0.000";
FCCWt2.Text = "0.000";

FCCWt3.Text $=" 0.000 "$;

FCCObR1.Text $=$ "0.000";

FCCObR2.Text $=$ "0.000";

FCCObR3.Text $=$ "0.000";

FCCActR1.Text = "0.000";

FCCActR2.Text = "0.000";

FCCActR3.Text = "0.000";

FCCCS1.Text $=" 0.000 "$;

FCCCS2.Text $=$ "0.000";

FCCCS3.Text $=" 0.000 "$;

FCCObRAvg.Text = "0.000";

FCCActRAvg.Text = "0.000";

FCCCSAvg.Text = "0.000";

If the user opts for storing non-confirming tests results private void CSRb28d_CheckedChanged(object sender, EventArgs e)

$$
\{
$$

CCCuringPeriod $=28$;

FCCC_B.Text = "28 ";

CCCGrade $=$ Convert. ToDouble $($ FCCGrade. Text $)$;

FCCC_A.Text $=$ CCCGrade.ToString();

\}

\}

Results are checked for respective grade and age of sample and its compressive strength value.

\} else if ((ChangeVal $==$ DialogResult.No $))$

MessageBox.Show("The Non Confirming Results will be

Stored");

private void FCCGrade_Leave(object sender, EventArgs e

CCCGrade $=$ Convert. ToDouble $($ FCCGrade. Text $) ;$

$$
\text { \} }
$$

Check for 7 days curing cube compressive strength

private void CSRb7d_CheckedChanged(object sender, EventArgs e)

CCCuringPeriod $=7$;

FCCC_B.Text = " ";

CCCGrade $=$ Convert.ToDouble $($ FCCGrade.Text $)$;

FCCC_A.Text $=$ CCCGrade.ToString();

FCCC_B.Text = "7";

CCCGradeP $=$ CCCGrade $* 0.67$;

FCCC_C.Text $=($ CCCGradeP.ToString ()$)$;

$$
\}
$$

Check for 28 days curing cube compressive strength

(4) Testing the Program.

Figure-3 shows data input and output of compressive strength with remarks of compressive strength after 28 days for M20 Grade of Concrete. The results are verified by manual calculations and found correct.

As per Figure-4 the user is given option either to change the non-confirming results with re-test, then he can opt to change the results by clicking yes. If option yes is given then 
all the values becomes zero and form becomes blank. If opted no then the non-confirming results are stored for records. The radio button provided on form either to select curing period of 7 days or 28 days and input of concrete grade governs the criteria for the conformity to the standards. If radio button 7 days is selected the Remarks at

Reterence Code Is 516-1959 Reamiremed 2013. Comprestre Streneth of Coarrete Cate:

\begin{tabular}{cc|}
$\qquad \begin{array}{c}\text { Sample ID } \\
\text { Mactine Calibration Constant. }\end{array}$ & 1.000
\end{tabular}

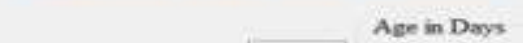

\begin{tabular}{|c|c|c|c|}
\hline \multirow{2}{*}{\multicolumn{2}{|c|}{ Concrete Grade M }} & \multicolumn{2}{|c|}{ Age in Days } \\
\hline & & O. Days & (4) 28 Days \\
\hline 1 & 2 & 3 & Average \\
\hline 150 & 148 & 149 & 149 \\
\hline 149 & 148 & 150 & 149 \\
\hline 150 & 150 & 150 & 150 \\
\hline 8500 & 8.540 & 8520 & 8.52 \\
\hline 2535.421 & 2599221 & 2541.387 & 2558.68 \\
\hline 500 & 450 & 480 & 47667 \\
\hline 500 & 450 & 480 & 476,67 \\
\hline 2237 & 20.54 & 21.48 & 21.46 \\
\hline
\end{tabular}

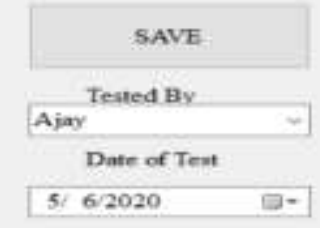

the bottom of the form changes to $0.67 *$ Grade of concrete selected and for selection of curing period of 28 days full compressive strength required as per norms is displayed at the bottom of the form.

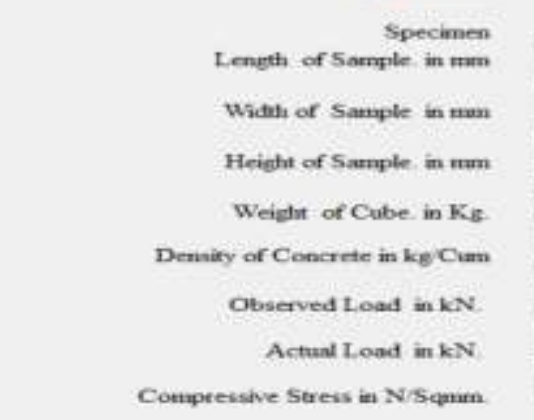

TRNMIRKS

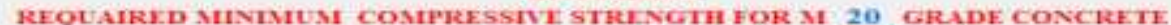
AT 28 DAVS AGE OFCONCRETE $=20$ NAqum.

Figure -3 Input and Output of Compressive Strength

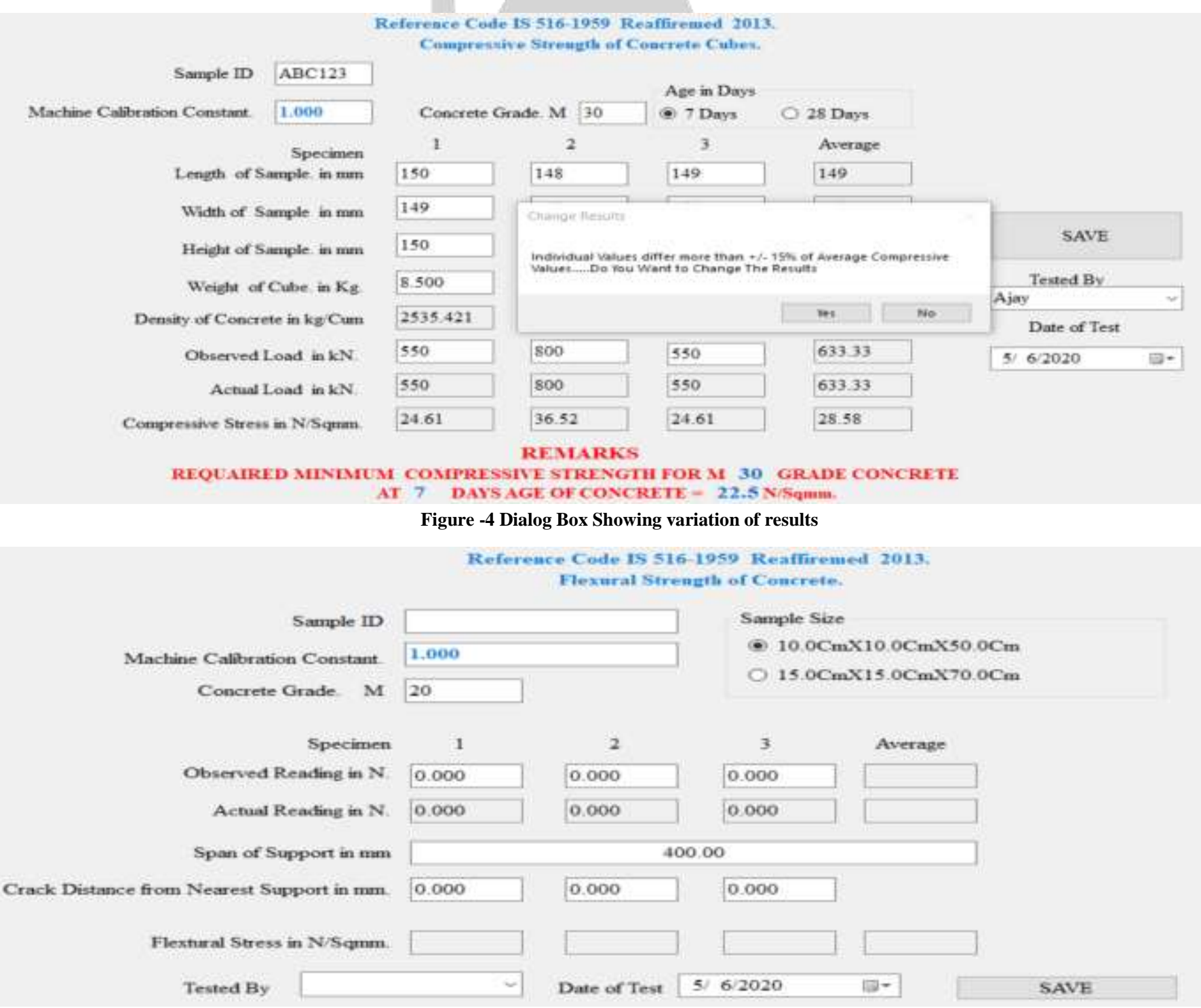

Figure -5 Flexural Strength Test Input form 
(B)

\section{Flexural Strength of Concrete}

The form for flexural strength calculations is designed as per Figure -5. The input data like Sample ID, Machine calibration constant, grade of concrete, onserved reading, span of support and crack distance to nearest support is given as input. The span input is restricted between 400 to $450 \mathrm{~mm}$ by putting if condition in the program. The selection of sample size either $10.0 \times 10.0 \times 50 \mathrm{~cm}$ or $15 \mathrm{x} .0 \mathrm{x} 15.0 \mathrm{x} 70.0 \mathrm{~cm}$ is done by clicking radio button The flexural strength as per the sample size selected on radio button and depending upon the distance of crack from nearest suport one of the two formula as per stated (1) or (2) is used and final flexural strengths are calculated as per Figure- 6 for three specimens.

\section{(5) Documentation.}

The Programs so prepared are cleaned for duplicate variables, intermediate checks and a neat \& clean and compact program stored in hard and soft copies.

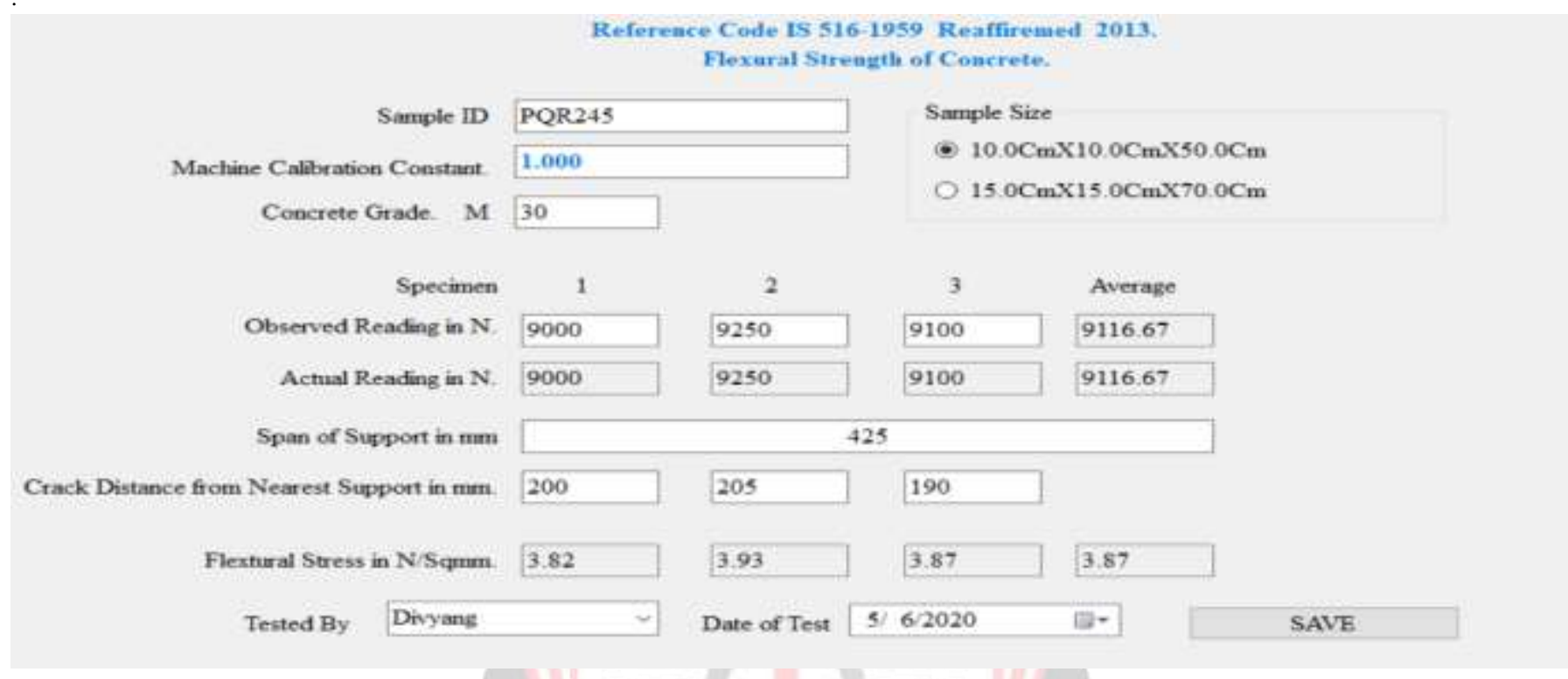

Figure -6 Flexural Strength Test output

\section{CONCLUSION}

With help of programing language a Graphic User Based program can be devised for two major properties of concrete (i) compressive strength and (ii) flexural strength of cocnrete. The program so prepared are standalone and do not requaire tools like MSExcel or any other cell based data sheet tools. The calculations formulea, validations for results, ranges for the inputs and results fixed once are cannot be changed or altered by the operator and thus the accuracy of the results is assured thorughout the use of the program. The results of compressive strength and flexural strength so obtained from the program are fast correct and accurate compared to other conventional tools. The results are stored as separated database and can be retrived as and when requaired with just reference to Poject Code Also other tests be programed and bunch of progams be prepared as software for civil engineering material testings.

\section{REFERENCES}

[1]. IS 516 : 1959 Reaffirmed 2013- METHODS OF TESTS FOR STRENGTH OF CONCRETE.

[2]. Microsoft Visual C\# step by step by John Sharp.

[3]. C\# Programming Yellow Book Rob Miles "Cheese" Edition 8.2 November 2016.
[4]. Beginners Guide to C\# and the .NET Micro Framework by GHI Electronics, LLC.

[5]. LEARNVERN An Android Based Application for computer programming.

[6]. Santosh G Gandevikar, Arjun M Butala, "Development of Computer Program for Calculating Shear Parameters of Soil", International Journal of Science and Research (IJSR), https://www.ijsr.net/search_index_results_paperid.p hp?id=SR20428095435, Volume 9 Issue 4, April 2020, 1716 $-1721$ 